\title{
A Density-Based Basis-Set Incompleteness Correction for GW Methods
}

\author{
Pierre-François Loos, ${ }^{1, \text { a) }}$ Barthélémy Pradines, ${ }^{2,3}$ Anthony Scemama, ${ }^{1}$ Emmanuel Giner, ${ }^{2}$ and Julien \\ Toulouse $2,4, \mathrm{~b})$ \\ ${ }^{1)}$ Laboratoire de Chimie et Physique Quantiques (UMR 5626), Université de Toulouse, CNRS, UPS, France \\ ${ }^{2)}$ Laboratoire de Chimie Théorique (UMR 7616), Sorbonne Université, CNRS, Paris, France \\ ${ }^{3)}$ Institut des Sciences du Calcul et des Données, Sorbonne Université, Paris, France \\ ${ }^{4)}$ Institut Universitaire de France, Paris, France
}

Similar to other electron correlation methods, many-
body perturbation theory methods based on Green func-
tions, such as the so-called $G W$ approximation, suffer
from the usual slow convergence of energetic properties
with respect to the size of the one-electron basis set.
This displeasing feature is due to the lack of explicit
electron-electron terms modeling the infamous Kato
electron-electron cusp and the correlation Coulomb
hole around it. Here, we propose a computationally
efficient density-based basis-set correction based on
short-range correlation density functionals which sig-
nificantly speeds up the convergence of energetics to-

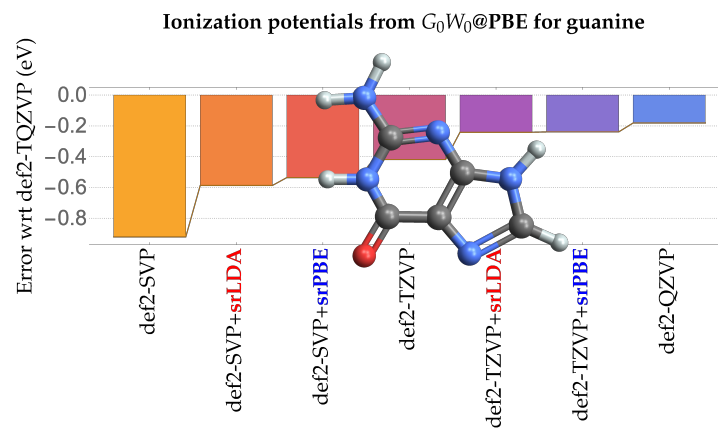

wards the complete basis set limit. The performance of this density-based correction is illustrated by computing the ionization potentials of the twenty smallest atoms and molecules of the GW100 test set at the perturbative $G W$ (or $G_{0} W_{0}$ ) level using increasingly large basis sets. We also compute the ionization potentials of the five canonical nucleobases (adenine, cytosine, thymine, guanine, and uracil) and show that, here again, a significant improvement is obtained.

\section{INTRODUCTION}

The purpose of many-body perturbation theory (MBPT) based on Green functions is to solve the formidable many-body problem by adding the electron-electron Coulomb interaction perturbatively starting from an independent-particle model. ${ }^{1}$ In this approach, the screening of the Coulomb interaction is an essential quantity. ${ }^{2-4}$

The so-called $G W$ approximation is the workhorse of MBPT and has a long and successful history in the calculation of the electronic structure of solids. ${ }^{2-4} G W$ is getting increasingly popular in molecular systems ${ }^{5-20}$ thanks to efficient implementation relying on plane waves ${ }^{21-23}$ or local basis functions. ${ }^{5,9,24-32}$ The $G W$ approximation stems from the acclaimed Hedin's equations ${ }^{33}$

$$
\begin{aligned}
G(12)= & G_{0}(12)+\int G_{0}(13) \Sigma(34) G(42) d(34) \\
\Gamma(123)= & \delta(12) \delta(13) \\
& +\int \frac{\delta \Sigma(12)}{\delta G(45)} G(46) G(75) \Gamma(673) d(4567), \\
P(12)= & -i \int G(13) G(41) \Gamma(342) d(34) \\
W(12)= & v(12)+\int v(13) P(34) W(42) d(34) \\
\Sigma(12)= & i \int G(13) W(14) \Gamma(324) d(34)
\end{aligned}
$$

\footnotetext{
${ }^{a)}$ Corresponding author: loos@irsamc.ups-tlse.fr

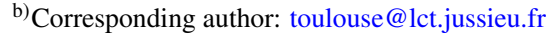

which connects the Green function $G$, its non-interacting version $G_{0}$, the irreducible vertex function $\Gamma$, the irreducible polarizability $P$, the dynamically-screened Coulomb interaction $W$ and the self-energy $\Sigma$, where $v$ is the bare Coulomb interaction, $\delta(12)$ is the Dirac delta function ${ }^{34}$ and 1 is a composite coordinate gathering space, spin, and time variables $\left(\mathbf{r}_{1}, \sigma_{1}, t_{1}\right)$. Within the $G W$ approximation, one bypasses the calculation of the vertex corrections by setting

$$
\Gamma(123) \stackrel{G W}{\approx} \delta(12) \delta(13) .
$$

Depending on the degree of self-consistency one is willing to perform, there exists several types of $G W$ calculations. ${ }^{35}$ The simplest and most popular variant of $G W$ is perturbative $G W$ (or $G_{0} W_{0}$ ). ${ }^{36,37}$ Although obviously starting-point dependent, ${ }^{38-40}$ it has been widely used in the literature to study solids, atoms, and molecules. ${ }^{7,16,17,38}$ For finite systems such as atoms and molecules, partially $5,6,37,41$ or fully self-consistent ${ }^{29-32} G W$ methods have shown great promise. $5,6,14,24,42-44$

Similar to other electron correlation methods, MBPT methods suffer from the usual slow convergence of energetic properties with respect to the size of the one-electron basis set. This can be tracked down to the lack of explicit electron-electron terms modeling the infamous electron-electron coalescence point (also known as Kato cusp ${ }^{45}$ ) and, more specifically, the Coulomb correlation hole around it. Pioneered by Hylleraas ${ }^{46}$ in the 1930's and popularized in the 1990's by Kutzelnigg and coworkers $^{47-49}$ (and subsequently others ${ }^{50-54}$ ), the so-called F12 methods overcome this slow convergence by employing geminal basis functions that closely resemble the correlation holes in electronic wave functions. F12 methods are now routinely employed in computational chemistry and provide robust 
tools for electronic structure calculations where small basis sets may be used to obtain near complete basis set (CBS) limit accuracy. ${ }^{55}$

The basis-set correction presented here follow a different route, and relies on the range-separated density-functional theory (RS-DFT) formalism to capture, thanks to a short-range correlation functional, the missing part of the short-range correlation effects. ${ }^{56}$ As shown in recent studies on both groundand excited-state properties, ${ }^{57,58}$ similar to F12 methods, it significantly speeds up the convergence of energetics towards the CBS limit while avoiding the usage of the large auxiliary basis sets that are used in F12 methods to avoid the numerous three- and four-electron integrals. ${ }^{50-54,59-61}$

Explicitly correlated F12 correction schemes have been derived for second-order Green function methods (GF2) ) $^{35,62-72}$ by Ten-no and coworkers ${ }^{73,74}$ and Valeev and coworkers. ${ }^{75,76}$ However, to the best of our knowledge, a F12-based correction for $G W$ has not been designed yet.

In the present manuscript, we illustrate the performance of the density-based basis-set correction developed in Refs. 56-58 on ionization potentials obtained within $G_{0} W_{0}$. Note that the present basis-set correction can be straightforwardly applied to other properties (e.g, electron affinities and fundamental gaps), as well as other flavors of (self-consistent) $G W$ or Green function-based methods, such as GF2 (and its higher-order variants).

The paper is organized as follows. In Sec. II, we provide details about the theory behind the present basis-set correction and its adaptation to $G W$ methods. Results for a large collection of molecular systems are reported and discussed in Sec. IV. Finally, we draw our conclusions in Sec. V. Unless otherwise stated, atomic units are used throughout.

\section{THEORY}

\section{A. MBPT with DFT basis-set correction}

Following Ref. 56, we start by defining, for a $N$-electron system with nuclei-electron potential $v_{\text {ne }}(\mathbf{r})$, the approximate ground-state energy for one-electron densities $n$ which are "representable" in a finite basis set $\mathcal{B}$

$$
E_{0}^{\mathcal{B}}=\min _{n \in \mathcal{D}^{\mathcal{B}}}\left\{F[n]+\int v_{\mathrm{ne}}(\mathbf{r}) n(\mathbf{r}) d \mathbf{r}\right\},
$$

where $\mathcal{D}^{\mathcal{B}}$ is the set of $N$-representable densities which can be extracted from a wave function $\Psi^{\mathcal{B}}$ expandable in the Hilbert space generated by $\mathcal{B}$. In this expression,

$$
F[n]=\min _{\Psi \rightsquigarrow n}\left\langle\Psi\left|\hat{T}+\hat{W}_{\mathrm{ee}}\right| \Psi\right\rangle
$$

is the exact Levy-Lieb universal density functional, ${ }^{77-79}$ where the notation $\Psi \rightsquigarrow n$ in Eq. (3) states that $\Psi$ yields the oneelectron density $n . \hat{T}$ and $\hat{W}_{\mathrm{ee}}$ are the kinetic and electronelectron interaction operators. The exact Levy-Lieb universal density functional is then decomposed as

$$
F[n]=F^{\mathcal{B}}[n]+\bar{E}^{\mathcal{B}}[n],
$$

where $F^{\mathcal{B}}[n]$ is the Levy-Lieb density functional with wave functions $\Psi^{\mathcal{B}}$ expandable in the Hilbert space generated by $\mathcal{B}$

$$
F^{\mathcal{B}}[n]=\min _{\Psi^{\mathcal{B}} \rightsquigarrow n}\left\langle\Psi^{\mathcal{B}}\left|\hat{T}+\hat{W}_{\mathrm{ee}}\right| \Psi^{\mathcal{B}}\right\rangle,
$$

and $\bar{E}^{\mathcal{B}}[n]$ is the complementary basis-correction density functional. ${ }^{56}$ In the present work, instead of using wavefunction methods for calculating $F^{\mathcal{B}}[n]$, we use Green-function methods. We assume that there exists a functional $\Omega^{\mathcal{B}}\left[G^{\mathcal{B}}\right]$ of $N$-representable one-electron Green functions $G^{\mathcal{B}}\left(\mathbf{r}, \mathbf{r}^{\prime}, \omega\right)$ representable in the basis set $\mathcal{B}$ and yielding the density $n$ which gives $F^{\mathcal{B}}[n]$ at a stationary point

$$
F^{\mathcal{B}}[n]=\operatorname{stat}_{G^{\mathcal{B}} \rightsquigarrow n} \Omega^{\mathcal{B}}\left[G^{\mathcal{B}}\right] .
$$

The reason why we use a stationary condition rather than a minimization condition is that only a stationary property is generally known for functionals of the Green function. For example, we can choose for $\Omega^{\mathcal{B}}[G]$ a Klein-like energy functional (see, e.g, Refs. 1, 65, 80-82)

$$
\Omega^{\mathcal{B}}[G]=\operatorname{Tr}[\ln (-G)]-\operatorname{Tr}\left[\left(G_{\mathrm{f}}^{\mathcal{B}}\right)^{-1} G-1\right]+\Phi_{\mathrm{Hxc}}^{\mathcal{B}}[G],
$$

where $\left(G_{\mathrm{f}}^{\mathcal{B}}\right)^{-1}$ is the projection into $\mathcal{B}$ of the inverse freeparticle Green function

$$
\left(G_{\mathrm{f}}\right)^{-1}\left(\mathbf{r}, \mathbf{r}^{\prime}, \omega\right)=\left(\omega+\frac{\nabla_{\mathbf{r}}^{2}}{2}\right) \delta\left(\mathbf{r}-\mathbf{r}^{\prime}\right),
$$

and we have introduced the trace

$$
\operatorname{Tr}[A B]=\int_{-\infty}^{+\infty} \frac{d \omega}{2 \pi i} e^{i \omega 0^{+}} \iint A\left(\mathbf{r}, \mathbf{r}^{\prime}, \omega\right) B\left(\mathbf{r}^{\prime}, \mathbf{r}, \omega\right) d \mathbf{r} d \mathbf{r}^{\prime} .
$$

In Eq. (8), $\Phi_{\mathrm{Hxc}}^{\mathcal{B}}[G]$ is a Hartree-exchange-correlation (Hxc) functional of the Green function such that its functional derivatives yields the Hxc self-energy in the basis

$$
\frac{\delta \Phi_{\mathrm{Hxc}}^{\mathcal{B}}[G]}{\delta G\left(\mathbf{r}, \mathbf{r}^{\prime}, \omega\right)}=\Sigma_{\mathrm{Hxc}}^{\mathcal{B}}[G]\left(\mathbf{r}, \mathbf{r}^{\prime}, \omega\right) \text {. }
$$

Inserting Eqs. (5) and (7) into Eq. (3), we finally arrive at

$$
E_{0}^{\mathcal{B}}=\operatorname{stat}_{G^{\mathcal{B}}}\left\{\Omega^{\mathcal{B}}\left[G^{\mathcal{B}}\right]+\int v_{\text {ne }}(\mathbf{r}) n_{G^{\mathcal{B}}}(\mathbf{r}) d \mathbf{r}+\bar{E}^{\mathcal{B}}\left[n_{G^{\mathcal{B}}}\right]\right\},
$$

where the stationary point is searched over $N$-representable one-electron Green functions $G^{\mathcal{B}}\left(\mathbf{r}, \mathbf{r}^{\prime}, \omega\right)$ representable in the basis set $\mathcal{B}$.

The stationary condition from Eq. (12) is

$$
\begin{aligned}
& \frac{\delta}{\delta G^{\mathcal{B}}}\left(\Omega^{\mathcal{B}}\left[G^{\mathcal{B}}\right]+\int v_{\mathrm{ne}}(\mathbf{r}) n_{G^{\mathcal{B}}}(\mathbf{r}) d \mathbf{r}+\bar{E}^{\mathcal{B}}\left[n_{G^{\mathcal{B}}}\right]\right. \\
&\left.-\lambda \int n_{G^{\mathcal{B}}}(\mathbf{r}) d \mathbf{r}\right)=0,
\end{aligned}
$$

where $\lambda$ is the chemical potential (enforcing the electron number). It leads the following Dyson equation

$$
\left(G^{\mathcal{B}}\right)^{-1}=\left(G_{0}^{\mathcal{B}}\right)^{-1}-\Sigma_{\mathrm{Hxc}}^{\mathcal{B}}\left[G^{\mathcal{B}}\right]-\bar{\Sigma}^{\mathcal{B}}\left[n_{G^{\mathcal{B}}}\right],
$$


where $\left(G_{0}^{\mathcal{B}}\right)^{-1}$ is the basis projection of the inverse noninteracting Green function with potential $v_{\text {ne }}(\mathbf{r})$, i.e,

$$
\left(G_{0}\right)^{-1}\left(\mathbf{r}, \mathbf{r}^{\prime}, \omega\right)=\left(\omega+\frac{\nabla_{\mathbf{r}}^{2}}{2}-v_{\mathrm{ne}}(\mathbf{r})+\lambda\right) \delta\left(\mathbf{r}-\mathbf{r}^{\prime}\right),
$$

and $\bar{\Sigma}^{\mathcal{B}}$ is a frequency-independent local self-energy coming from the functional derivative of the complementary basiscorrection density functional

$$
\bar{\Sigma}^{\mathcal{B}}[n]\left(\mathbf{r}, \mathbf{r}^{\prime}\right)=\bar{v}^{\mathcal{B}}[n](\mathbf{r}) \delta\left(\mathbf{r}-\mathbf{r}^{\prime}\right),
$$

with $\bar{v}^{\mathcal{B}}[n](\mathbf{r})=\delta \bar{E}^{\mathcal{B}}[n] / \delta n(\mathbf{r})$. This is found from Eq. (13) by using the chain rule,

$$
\frac{\delta \bar{E}^{\mathcal{B}}[n]}{\delta G\left(\mathbf{r}, \mathbf{r}^{\prime}, \omega\right)}=\int \frac{\delta \bar{E}^{\mathcal{B}}[n]}{\delta n\left(\mathbf{r}^{\prime \prime}\right)} \frac{\delta n\left(\mathbf{r}^{\prime \prime}\right)}{\delta G\left(\mathbf{r}, \mathbf{r}^{\prime}, \omega\right)} d \mathbf{r}^{\prime \prime},
$$

and

$$
n(\mathbf{r})=\int_{-\infty}^{+\infty} \frac{d \omega}{2 \pi i} e^{i \omega 0^{+}} G(\mathbf{r}, \mathbf{r}, \omega) .
$$

The solution of the Dyson equation (14) gives the Green function $G^{\mathcal{B}}\left(\mathbf{r}, \mathbf{r}^{\prime}, \omega\right)$ which is not exact (even using the exact complementary basis-correction density functional $\bar{\Sigma}^{\mathcal{B}}[n]$ ) but should converge more rapidly with the basis set thanks to the presence of the basis-set correction $\bar{\Sigma}^{\mathcal{B}}$. Of course, in the CBS limit, the basis-set correction vanishes and the Green function becomes exact, i.e,

$$
\lim _{\mathcal{B} \rightarrow \mathrm{CBS}} \bar{\Sigma}^{\mathcal{B}}=0, \quad \quad \lim _{\mathcal{B} \rightarrow \mathrm{CBS}} G^{\mathcal{B}}=G .
$$

The Dyson equation (14) can also be written with an arbitrary reference

$$
\left(G^{\mathcal{B}}\right)^{-1}=\left(G_{\text {ref }}^{\mathcal{B}}\right)^{-1}-\left(\Sigma_{\mathrm{Hxc}}^{\mathcal{B}}\left[G^{\mathcal{B}}\right]-\Sigma_{\text {ref }}^{\mathcal{B}}\right)-\bar{\Sigma}^{\mathcal{B}}\left[n_{G^{\mathcal{B}}}\right],
$$

where $\left(G_{\text {ref }}^{\mathcal{B}}\right)^{-1}=\left(G_{0}^{\mathcal{B}}\right)^{-1}-\Sigma_{\text {ref }}^{\mathcal{B}}$. For example, if the reference is Hartree-Fock $(\mathrm{HF}), \Sigma_{\text {ref }}^{\mathcal{B}}\left(\mathbf{r}, \mathbf{r}^{\prime}\right)=\Sigma_{\mathrm{Hx}}^{\mathcal{B}}\left(\mathbf{r}, \mathbf{r}^{\prime}\right)$ is the HF nonlocal self-energy, and if the reference is Kohn-Sham (KS), $\Sigma_{\text {ref }}^{\mathcal{B}}\left(\mathbf{r}, \mathbf{r}^{\prime}\right)=v_{\mathrm{Hxc}}^{\mathcal{B}}(\mathbf{r}) \delta\left(\mathbf{r}-\mathbf{r}^{\prime}\right)$ is the local Hxc potential.

Note that the present basis-set correction is applicable to any approximation of the self-energy (irrespectively of the diagrams included) without altering the CBS limit of such methods. Consequently, it can be applied, for example, to GF2 methods (also known as second Born approximation ${ }^{65}$ in the condensed-matter community) or higher orders. ${ }^{35,62-72}$ Note, however, that the basis-set correction is optimal for the exact self-energy within a given basis set, since it corrects only for the basis-set error and not for the chosen approximate form of the self-energy within the basis set.

\section{B. The $G W$ Approximation}

In this subsection, we provide the minimal set of equations required to describe $G_{0} W_{0}$. More details can be found, for example, in Refs. 9,25,27. For the sake of simplicity, we only give the equations for closed-shell systems with a KS singleparticle reference (with a local potential). The one-electron energies $\epsilon_{p}$ and their corresponding (real-valued) orbitals $\phi_{p}(\mathbf{r})$ (which defines the basis set $\mathcal{B}$ ) are then the KS orbitals and their orbital energies.

Within the $G W$ approximation, the correlation part of the self-energy reads

$$
\begin{aligned}
\Sigma_{\mathrm{c}, p}^{\mathcal{B}}(\omega) & =\left\langle\phi_{p}\left|\Sigma_{\mathrm{c}}^{\mathcal{B}}(\omega)\right| \phi_{p}\right\rangle \\
& =2 \sum_{i}^{N_{\text {occ }}} \sum_{m} \frac{[p i \mid m]^{2}}{\omega-\epsilon_{i}+\Omega_{m}-i \eta} \\
& +2 \sum_{a}^{N_{\text {virt }}} \sum_{m} \frac{[p a \mid m]^{2}}{\omega-\epsilon_{a}-\Omega_{m}+i \eta},
\end{aligned}
$$

where $i$ runs over the $N_{\text {occ }}$ occupied orbitals, $a$ runs over the $N_{\text {virt }}$ virtual orbitals, $m$ labels excited states (see below), and $\eta$ is a positive infinitesimal. The screened two-electron integrals

$$
[p q \mid m]=\sum_{i}^{N_{\text {occ }}} \sum_{a}^{N_{\text {virt }}}(p q \mid i a)\left(\boldsymbol{X}_{m}+\boldsymbol{Y}_{m}\right)_{i a}
$$

are obtained via the contraction of the bare two-electron integrals $^{83}$

$$
(p q \mid r s)=\iint \frac{\phi_{p}(\mathbf{r}) \phi_{q}(\mathbf{r}) \phi_{r}\left(\mathbf{r}^{\prime}\right) \phi_{s}\left(\mathbf{r}^{\prime}\right)}{\left|\mathbf{r}-\mathbf{r}^{\prime}\right|} d \mathbf{r} d \mathbf{r}^{\prime},
$$

and the transition densities $\left(\boldsymbol{X}_{m}+\boldsymbol{Y}_{m}\right)_{i a}$ originating from a (direct) random-phase approximation (RPA) calculation ${ }^{84,85}$

$$
\left(\begin{array}{cc}
\boldsymbol{A} & \boldsymbol{B} \\
-\boldsymbol{B} & -\boldsymbol{A}
\end{array}\right)\left(\begin{array}{c}
\boldsymbol{X}_{m} \\
\boldsymbol{Y}_{m}
\end{array}\right)=\Omega_{m}\left(\begin{array}{c}
\boldsymbol{X}_{m} \\
\boldsymbol{Y}_{m}
\end{array}\right)
$$

with

$$
A_{i a, j b}=\delta_{i j} \delta_{a b}\left(\epsilon_{a}-\epsilon_{i}\right)+2(i a \mid b j), \quad B_{i a, j b}=2(i a \mid j b),
$$

and $\delta_{p q}$ is the Kronecker delta. ${ }^{34}$ Equation (24) also provides the RPA neutral excitation energies $\Omega_{m}$ which correspond to the poles of the screened Coulomb interaction $W(\omega)$.

The $G_{0} W_{0}$ quasiparticle energies $\epsilon_{p}^{G_{0} W_{0}}$ are provided by the solution of the (non-linear) quasiparticle equation ${ }^{25,36,86}$

$$
\omega=\epsilon_{p}-V_{\mathrm{xc}, p}^{\mathcal{B}}+\Sigma_{\mathrm{x}, p}^{\mathcal{B}}+\operatorname{Re}\left[\Sigma_{\mathrm{c}, p}^{\mathcal{B}}(\omega)\right] .
$$

with the largest renormalization weight (or factor)

$$
Z_{p}=\left[1-\left.\frac{\partial \operatorname{Re}\left[\Sigma_{\mathrm{c}, p}^{\mathcal{B}}(\omega)\right]}{\partial \omega}\right|_{\omega=\epsilon_{p}}\right]^{-1} .
$$

Because of sum rules, ${ }^{87-90}$ the other solutions, known as satellites, share the remaining weight. In Eq. (26), $\Sigma_{\mathrm{x}, p}^{\mathcal{B}}=$ $\left\langle\phi_{p}\left|\Sigma_{\mathrm{x}}^{\mathcal{B}}\right| \phi_{p}\right\rangle$ is the (static) HF exchange part of the self-energy and

$$
V_{\mathrm{xc}, p}^{\mathcal{B}}=\int \phi_{p}(\mathbf{r}) v_{\mathrm{xc}}^{\mathcal{B}}(\mathbf{r}) \phi_{p}(\mathbf{r}) d \mathbf{r},
$$


where $v_{\mathrm{xc}}^{\mathcal{B}}(\mathbf{r})$ is the KS exchange-correlation potential. In particular, the ionization potential (IP) and electron affinity (EA) are extracted thanks to the following relationships: ${ }^{62}$

$$
\mathrm{IP}=-\epsilon_{\mathrm{HOMO}}^{G_{0} W_{0}}, \quad \mathrm{EA}=-\epsilon_{\mathrm{LUMO}}^{G_{0} W_{0}},
$$

where $\epsilon_{\mathrm{HOMO}}^{G_{0} W_{0}}$ and $\epsilon_{\mathrm{LUMO}}^{G_{0} W_{0}}$ are the HOMO and LUMO quasiparticle energies, respectively.

\section{Basis-set correction}

The fundamental idea behind the present basis-set correction is to recognize that the singular two-electron Coulomb interaction $\left|\mathbf{r}-\mathbf{r}^{\prime}\right|^{-1}$ projected in a finite basis $\mathcal{B}$ is a finite, non-divergent quantity at $\left|\mathbf{r}-\mathbf{r}^{\prime}\right|=0$, which "resembles" the long-range interaction operator $\left|\mathbf{r}-\mathbf{r}^{\prime}\right|^{-1} \operatorname{erf}\left(\mu\left|\mathbf{r}-\mathbf{r}^{\prime}\right|\right)$ used within RS-DFT. ${ }^{56}$

We start therefore by considering an effective non-divergent two-electron interaction $W^{\mathcal{B}}\left(\mathbf{r}, \mathbf{r}^{\prime}\right)$ within the basis set which reproduces the expectation value of the Coulomb interaction over a given pair density $n_{2}^{\mathcal{B}}\left(\mathbf{r}, \mathbf{r}^{\prime}\right)$, i.e,

$$
\frac{1}{2} \iint \frac{n_{2}^{\mathcal{B}}\left(\mathbf{r}, \mathbf{r}^{\prime}\right)}{\left|\mathbf{r}-\mathbf{r}^{\prime}\right|} d \mathbf{r} d \mathbf{r}^{\prime}=\frac{1}{2} \iint n_{2}^{\mathcal{B}}\left(\mathbf{r}, \mathbf{r}^{\prime}\right) W^{\mathcal{B}}\left(\mathbf{r}, \mathbf{r}^{\prime}\right) d \mathbf{r} d \mathbf{r}^{\prime}
$$

The properties of $W^{\mathcal{B}}\left(\mathbf{r}, \mathbf{r}^{\prime}\right)$ are detailed in Ref. 56. A key aspect is that because the value of $W^{\mathcal{B}}\left(\mathbf{r}, \mathbf{r}^{\prime}\right)$ at coalescence, $W^{\mathcal{B}}(\mathbf{r}, \mathbf{r})$, is necessarily finite in a finite basis $\mathcal{B}$, one can approximate $W^{\mathcal{B}}\left(\mathbf{r}, \mathbf{r}^{\prime}\right)$ by a non-divergent, long-range interaction of the form

$$
W^{\mathcal{B}}\left(\mathbf{r}, \mathbf{r}^{\prime}\right) \approx \frac{1}{2}\left\{\frac{\operatorname{erf}\left[\mu^{\mathcal{B}}(\mathbf{r})\left|\mathbf{r}-\mathbf{r}^{\prime}\right|\right]}{\left|\mathbf{r}-\mathbf{r}^{\prime}\right|}+\frac{\operatorname{erf}\left[\mu^{\mathcal{B}}\left(\mathbf{r}^{\prime}\right)\left|\mathbf{r}-\mathbf{r}^{\prime}\right|\right]}{\left|\mathbf{r}-\mathbf{r}^{\prime}\right|}\right\} .
$$

The information about the finiteness of the basis set is then transferred to the range-separation function $\mu^{\mathcal{B}}(\mathbf{r})$, and its value can be determined by ensuring that the two sides of Eq. (31) are strictly equal at $\left|\mathbf{r}-\mathbf{r}^{\prime}\right|=0$. Knowing that $\lim _{r \rightarrow 0} \operatorname{erf}(\mu r) / r=$ $2 \mu / \sqrt{\pi}$, this yields

$$
\mu^{\mathcal{B}}(\mathbf{r})=\frac{\sqrt{\pi}}{2} W^{\mathcal{B}}(\mathbf{r}, \mathbf{r})
$$

Following Refs. 56-58, we adopt the following definition for $W^{\mathcal{B}}\left(\mathbf{r}, \mathbf{r}^{\prime}\right)$

$$
W^{\mathcal{B}}\left(\mathbf{r}, \mathbf{r}^{\prime}\right)= \begin{cases}f^{\mathcal{B}}\left(\mathbf{r}, \mathbf{r}^{\prime}\right) / n_{2}^{\mathcal{B}}\left(\mathbf{r}, \mathbf{r}^{\prime}\right), & \text { if } n_{2}^{\mathcal{B}}\left(\mathbf{r}, \mathbf{r}^{\prime}\right) \neq 0 \\ \infty, & \text { otherwise }\end{cases}
$$

where, in this work, $f^{\mathcal{B}}\left(\mathbf{r}, \mathbf{r}^{\prime}\right)$ and $n_{2}^{\mathcal{B}}\left(\mathbf{r}, \mathbf{r}^{\prime}\right)$ are calculated using the opposite-spin two-electron density matrix of a spinrestricted single determinant (such as HF and KS). For a closedshell system, we have

$$
f^{\mathcal{B}}\left(\mathbf{r}, \mathbf{r}^{\prime}\right)=2 \sum_{p q}^{N_{\text {bas }}} \sum_{i j}^{N_{\text {occ }}} \phi_{p}(\mathbf{r}) \phi_{i}(\mathbf{r})(p i \mid q j) \phi_{q}\left(\mathbf{r}^{\prime}\right) \phi_{j}\left(\mathbf{r}^{\prime}\right),
$$

and

$$
n_{2}^{\mathcal{B}}\left(\mathbf{r}, \mathbf{r}^{\prime}\right)=2 \sum_{i j}^{N_{\text {occ }}} \phi_{i}(\mathbf{r})^{2} \phi_{j}\left(\mathbf{r}^{\prime}\right)^{2}=\frac{1}{2} n^{\mathcal{B}}(\mathbf{r}) n^{\mathcal{B}}\left(\mathbf{r}^{\prime}\right),
$$

where $n^{\mathcal{B}}(\mathbf{r})$ is the one-electron density. The quantity $n_{2}^{\mathcal{B}}\left(\mathbf{r}, \mathbf{r}^{\prime}\right)$ represents the opposite-spin pair density of a closed-shell system with a single-determinant wave function. Note that in Eq. (34) the indices $p$ and $q$ run over all occupied and virtual orbitals ( $N_{\text {bas }}=N_{\text {occ }}+N_{\text {virt }}$ is the total dimension of the basis set).

Thanks to this definition, the effective interaction $W^{\mathcal{B}}\left(\mathbf{r}, \mathbf{r}^{\prime}\right)$ has the interesting property

$$
\lim _{\mathcal{B} \rightarrow \mathrm{CBS}} W^{\mathcal{B}}\left(\mathbf{r}, \mathbf{r}^{\prime}\right)=\left|\mathbf{r}-\mathbf{r}^{\prime}\right|^{-1},
$$

which means that in the CBS limit one recovers the genuine (divergent) Coulomb interaction. Therefore, in the CBS limit, the coalescence value $W^{\mathcal{B}}(\mathbf{r}, \mathbf{r})$ goes to infinity, and so does $\mu^{\mathcal{B}}(\mathbf{r})$. Since the present basis-set correction employs complementary short-range correlation potentials from RS-DFT which have the property of going to zero when $\mu$ goes to infinity, the present basis-set correction properly vanishes in the CBS limit.

\section{Short-range correlation functionals}

The frequency-independent local self-energy $\bar{\Sigma}^{\mathcal{B}}[n]\left(\mathbf{r}, \mathbf{r}^{\prime}\right)=$ $\bar{v}^{\mathcal{B}}[n](\mathbf{r}) \delta\left(\mathbf{r}-\mathbf{r}^{\prime}\right)$ originates from the functional derivative of complementary basis-correction density functionals $\bar{v}^{\mathcal{B}}[n](\mathbf{r})=$ $\delta \bar{E}^{\mathcal{B}}[n] / \delta n(\mathbf{r})$.

In this work, we have tested two complementary density functionals coming from two approximations to the short-range correlation functional with multideterminant (md) reference of RS-DFT. ${ }^{91}$ The first one is a short-range local-density approximation $(\text { srLDA })^{91,92}$

$$
\bar{E}_{\mathrm{srLDA}}^{\mathcal{B}}[n]=\int n(\mathbf{r}) \bar{\varepsilon}_{\mathrm{c}, \mathrm{md}}^{\mathrm{srLDA}}\left(n(\mathbf{r}), \mu^{\mathcal{B}}(\mathbf{r})\right) d \mathbf{r},
$$

where the correlation energy per particle $\bar{\varepsilon}_{\mathrm{c}, \mathrm{md}}^{\mathrm{srLD}}(n, \mu)$ has been parametrized from calculations on the uniform electron gas ${ }^{93}$ reported in Ref. 92. The second one is a short-range PerdewBurke-Ernzerhof (srPBE) approximation ${ }^{57,94}$

$$
\bar{E}_{\mathrm{srPBE}}^{\mathcal{B}}[n]=\int n(\mathbf{r}) \bar{\varepsilon}_{\mathrm{c}, \mathrm{md}}^{\mathrm{srBE}}\left(n(\mathbf{r}), s(\mathbf{r}), \mu^{\mathcal{B}}(\mathbf{r})\right) d \mathbf{r},
$$

where $s(\mathbf{r})=\nabla n(\mathbf{r}) / n(\mathbf{r})^{4 / 3}$ is the reduced density gradient and the correlation energy per particle $\bar{\varepsilon}_{\mathrm{c}, \mathrm{md}}^{\mathrm{srPB}}(n, s, \mu)$ interpolates between the usual PBE correlation energy per particle ${ }^{95}$ at $\mu=$ 0 and the exact large- $\mu$ behavior ${ }^{92,96,97}$ using the on-top pair density of the Coulombic uniform electron gas (see Ref. 57). Note that the information on the local basis-set incompleteness error is provided to these RS-DFT functionals through the range-separation function $\mu^{\mathcal{B}}(\mathbf{r})$.

From these energy functionals, we generate the potentials $\bar{v}_{\mathrm{srLDA}}^{\mathcal{B}}[n](\mathbf{r})=\delta \bar{E}_{\mathrm{srLDA}}^{\mathcal{B}}[n] / \delta n(\mathbf{r})$ and $\bar{v}_{\mathrm{srPBE}}^{\mathcal{B}}[n](\mathbf{r})=$ 
$\delta \bar{E}_{\text {srPBE }}^{\mathcal{B}}[n] / \delta n(\mathbf{r})$ (considering $\mu^{\mathcal{B}}(\mathbf{r})$ as being fixed) which are then used to obtain the basis-set corrected $G_{0} W_{0}$ quasiparticle energies

$$
\bar{\epsilon}_{p}^{G_{0} W_{0}}=\epsilon_{p}^{G_{0} W_{0}}+\bar{V}_{p}^{\mathcal{B}}
$$

with

$$
\bar{V}_{p}^{\mathcal{B}}=\int \phi_{p}(\mathbf{r}) \bar{v}^{\mathcal{B}}[n](\mathbf{r}) \phi_{p}(\mathbf{r}) d \mathbf{r}
$$

where $\bar{v}^{\mathcal{B}}[n](\mathbf{r})=\bar{v}_{\text {srLDA }}^{\mathcal{B}}[n](\mathbf{r})$ or $\bar{v}_{\text {srPBE }}^{\mathcal{B}}[n](\mathbf{r})$ and the density is calculated from the HF or KS orbitals. The expressions of these srLDA and srPBE correlation potentials are provided in the supporting information.

As evidenced by Eq. (39), the present basis-set correction is a non-self-consistent, post-GW correction. Although outside the scope of this study, various other strategies can be potentially designed, for example, within linearized $G_{0} W_{0}$ or self-consistent $G W$ calculations.

\section{COMPUTATIONAL DETAILS}

All the geometries have been extracted from the GW100 set. ${ }^{16}$ Unless otherwise stated, all the $G_{0} W_{0}$ calculations have been performed with the MOLGW software developed by Bruneval and coworkers. ${ }^{10}$ The HF, PBE, and PBE0 calculations as well as the srLDA and srPBE basis-set corrections have been computed with Quantum Package,${ }^{98}$ which by default uses the SG-2 quadrature grid for the numerical integrations. Frozen-core (FC) calculations are systematically performed. The FC density-based basis-set correction ${ }^{57}$ is used consistently with the FC approximation in the $G_{0} W_{0}$ calculations. The $G_{0} W_{0}$ quasiparticle energies have been obtained "graphically", i.e, by solving the non-linear, frequency-dependent quasiparticle equation (26) (without linearization). Moreover, the infinitesimal $\eta$ in Eq. (21) has been set to zero.

Compared to the conventional $O\left(N_{\text {occ }}^{3} N_{\text {virt }}^{3}\right)$ computational cost of $G W$, the present basis-set correction represents a marginal $O\left(N_{\text {occ }}^{2} N_{\text {bas }}^{2} N_{\text {grid }}\right)$ additional cost as further discussed in Refs. 57 and 58. Note, however, that the formal $O\left(N_{\text {occ }}^{3} N_{\text {virt }}^{3}\right)$ computational scaling of $G W$ can be significantly reduced thanks to resolution-of-the-identity techniques ${ }^{9,25,99}$ and other tricks. ${ }^{100,101}$

\section{RESULTS AND DISCUSSION}

In this section, we study a subset of atoms and molecules from the GW100 test set. ${ }^{16}$ In particular, we study the 20 smallest molecules of the GW100 set, a subset that we label as GW20. This subset has been recently considered by Lewis and Berkelbach to study the effect of vertex corrections to $W$ on IPs of molecules. ${ }^{103}$ Later in this section, we also study the five canonical nucleobases (adenine, cytosine, thymine, guanine, and uracil) which are also part of the GW100 test set.

\section{A. GW20}

The IPs of the GW20 set obtained at the $G_{0} W_{0} @ \mathrm{HF}$ and $G_{0} W_{0} @$ PBE0 levels with increasingly larger Dunning's basis sets cc-pVXZ (X = D, T, Q, and 5) are reported in Tables I and II, respectively. The corresponding statistical deviations (with respect to the CBS values) are also reported: mean absolute deviation (MAD), root-mean-square deviation (RMSD), and maximum deviation (MAX). These reference CBS values have been obtained with the usual $\mathrm{X}^{-3}$ extrapolation procedure using the three largest basis sets.?

The convergence of the IP of the water molecule with respect to the basis set size is depicted in Fig. 1. This represents a typical example. Additional graphs reporting the convergence of the IPs of each molecule of the GW20 subset at the $G_{0} W_{0} @ \mathrm{HF}$ and $G_{0} W_{0} @ \mathrm{PBE} 0$ levels are reported in the supporting information.

Tables I and II (as well as Fig. 1) clearly evidence that the present basis-set correction significantly increases the rate of convergence of IPs. At the $G_{0} W_{0} @ \mathrm{HF}$ (see Table I), the MAD of the conventional calculations (i.e, without basis-set correction) is roughly divided by two each time one increases the basis set size (MADs of 0.60, 0.24, 0.10 , and $0.05 \mathrm{eV}$ going from cc-pVDZ to cc-pV5Z) with maximum errors higher than $1 \mathrm{eV}$ for molecules such as $\mathrm{HF}, \mathrm{H}_{2} \mathrm{O}$, and $\mathrm{LiF}$ with the smallest basis set. Even with the largest quintuple- $\zeta$ basis, the MAD is still above chemical accuracy (i.e, error below $1 \mathrm{kcal} / \mathrm{mol}$ or $0.043 \mathrm{eV}$ ).

For each basis set, the correction brought by the short-range correlation functionals reduces by roughly half or more the MAD, RMSD, and MAX compared to the correction-free calculations. For example, we obtain MADs of $0.27,0.12,0.04$, and $0.01 \mathrm{eV}$ at the $G_{0} W_{0} @ \mathrm{HF}+\mathrm{srPBE}$ level with increasingly larger basis sets. Interestingly, in most cases, the srPBE correction is slightly larger than the srLDA one. This observation is clear at the cc-pVDZ level but, for larger basis sets, the two RS-DFT-based corrections are essentially equivalent. Note also that, in some cases, the corrected IPs slightly overshoot the CBS values. However, it is hard to know if it is not due to the extrapolation error. In a nutshell, the present basis-set correction provides cc-pVQZ quality results at the cc-pVTZ level. Besides, it allows to reach chemical accuracy with the quadruple- $\zeta$ basis set, an accuracy that could not be reached even with the cc-pV5Z basis set for the conventional calculations.

Very similar conclusions are drawn at the $G_{0} W_{0} @$ PBE0 level (see Table II) with a slightly faster convergence to the CBS limit. For example, at the $G_{0} W_{0} @ \mathrm{PBE} 0+$ srLDA/ccpVQZ level, the MAD is only $0.02 \mathrm{eV}$ with a maximum error as small as $0.09 \mathrm{eV}$.

It is worth pointing out that, for ground-state properties such as atomization and correlation energies, the density-based correction brought a larger acceleration of the basis-set convergence. For example, we evidenced in Ref. 57 that quintuple- $\zeta$ quality atomization and correlation energies are recovered with triple- $\zeta$ basis sets. Here, the overall gain seems to be less important. The possible reasons for this could be: i) DFT approximations are usually less accurate for the potential than 
TABLE I. IPs (in eV) of the 20 smallest molecules of the GW100 set computed at the $G_{0} W_{0} @ \mathrm{HF}$ level of theory with various basis sets and corrections. The mean absolute deviation (MAD), root-mean-square deviation (RMSD), and maximum deviation (MAX) with respect to the $G_{0} W_{0} @ \mathrm{HF} / \mathrm{CBS}$ values are also reported.

\begin{tabular}{|c|c|c|c|c|c|c|c|c|c|c|c|c|c|}
\hline \multirow[b]{2}{*}{ Mol. } & \multicolumn{4}{|c|}{$G_{0} W_{0} @ \mathrm{HF}$} & \multicolumn{4}{|c|}{$G_{0} W_{0} @ \mathrm{HF}+$ srLDA } & \multicolumn{4}{|c|}{$G_{0} W_{0} @ \mathrm{HF}+\mathrm{srPBE}$} & \multirow{2}{*}{$\frac{G_{0} W_{0} @ \mathrm{HF}}{\mathrm{CBS}}$} \\
\hline & cc-pVDZ & cc-pVTZ & cc-pVQZ & cc-pV5Z & cc-pVDZ & cc-pVTZ & cc-pVQZ & cc-pV5Z & cc-pVDZ & cc-pVTZ & cc-pVQZ & cc-pV5Z & \\
\hline$\overline{\mathrm{He}}$ & 24.36 & 24.57 & 24.67 & 24.72 & 24.63 & 24.69 & 24.73 & 24.74 & 24.66 & 24.69 & 24.72 & 24.74 & 24.75 \\
\hline $\mathrm{Ne}$ & 20.87 & 21.39 & 21.63 & 21.73 & 21.38 & 21.67 & 21.80 & 21.84 & 21.56 & 21.73 & 21.81 & 21.83 & 21.82 \\
\hline $\mathrm{H}_{2}$ & 16.25 & 16.48 & 16.56 & 16.58 & 16.42 & 16.54 & 16.58 & 16.60 & 16.42 & 16.53 & 16.58 & 16.60 & 16.61 \\
\hline $\mathrm{Li}_{2}$ & 5.23 & 5.34 & 5.39 & 5.42 & 5.31 & 5.37 & 5.41 & 5.43 & 5.28 & 5.37 & 5.41 & 5.43 & 5.44 \\
\hline $\mathrm{LiH}$ & 7.96 & 8.16 & 8.25 & 8.28 & 8.13 & 8.23 & 8.28 & 8.30 & 8.10 & 8.21 & 8.27 & 8.30 & 8.31 \\
\hline $\mathrm{HF}$ & 15.54 & 16.16 & 16.42 & 16.52 & 16.01 & 16.41 & 16.57 & 16.61 & 16.15 & 16.45 & 16.57 & 16.61 & 16.62 \\
\hline $\mathrm{Ar}$ & 15.40 & 15.72 & 15.93 & 16.08 & 15.85 & 15.98 & 16.09 & 16.18 & 15.91 & 15.99 & 16.08 & 16.17 & 16.15 \\
\hline $\mathrm{H}_{2} \mathrm{O}$ & 12.16 & 12.79 & 13.04 & 13.14 & 12.58 & 13.01 & 13.16 & 13.21 & 12.68 & 13.03 & 13.16 & 13.20 & 13.23 \\
\hline $\mathrm{LiF}$ & 10.75 & 11.35 & 11.59 & 11.70 & 11.21 & 11.60 & 11.73 & 11.79 & 11.34 & 11.63 & 11.73 & 11.78 & 11.79 \\
\hline $\mathrm{HCl}$ & 12.40 & 12.77 & 12.96 & 13.05 & 12.79 & 12.99 & 13.10 & 13.13 & 12.83 & 12.99 & 13.09 & 13.12 & 13.12 \\
\hline $\mathrm{BeO}$ & 9.47 & 9.77 & 9.98 & 10.09 & 9.85 & 9.97 & 10.09 & 10.15 & 9.93 & 9.98 & 10.08 & 10.15 & 10.16 \\
\hline $\mathrm{CO}$ & 14.66 & 15.02 & 15.17 & 15.24 & 14.99 & 15.18 & 15.26 & 15.29 & 15.04 & 15.18 & 15.25 & 15.29 & 15.30 \\
\hline $\mathrm{N}_{2}$ & 15.87 & 16.31 & 16.48 & 16.56 & 16.22 & 16.50 & 16.59 & 16.62 & 16.30 & 16.50 & 16.58 & 16.62 & 16.62 \\
\hline $\mathrm{CH}_{4}$ & 14.43 & 14.74 & 14.86 & 14.90 & 14.69 & 14.85 & 14.91 & 14.93 & 14.73 & 14.85 & 14.90 & 14.93 & 14.95 \\
\hline $\mathrm{BH}_{3}$ & 13.35 & 13.64 & 13.74 & 13.78 & 13.57 & 13.73 & 13.78 & 13.80 & 13.58 & 13.72 & 13.78 & 13.80 & 13.82 \\
\hline $\mathrm{NH}_{3}$ & 10.59 & 11.13 & 11.32 & 11.40 & 10.93 & 11.30 & 11.41 & 11.45 & 10.99 & 11.30 & 11.41 & 11.44 & 11.47 \\
\hline $\mathrm{BF}$ & 11.08 & 11.30 & 11.38 & 11.42 & 11.29 & 11.40 & 11.43 & 11.45 & 11.29 & 11.38 & 11.42 & 11.45 & 11.45 \\
\hline $\mathrm{BN}$ & 11.35 & 11.69 & 11.85 & 11.92 & 11.67 & 11.85 & 11.94 & 11.98 & 11.72 & 11.85 & 11.93 & 11.97 & 11.98 \\
\hline $\mathrm{SH}_{2}$ & 10.10 & 10.49 & 10.65 & 10.72 & 10.44 & 10.67 & 10.76 & 10.78 & 10.45 & 10.66 & 10.74 & 10.77 & 10.78 \\
\hline $\mathrm{F}_{2}$ & 15.93 & 16.30 & 16.51 & 16.61 & 16.42 & 16.56 & 16.67 & 16.71 & 16.58 & 16.61 & 16.67 & 16.71 & 16.69 \\
\hline MAD & 0.66 & 0.30 & 0.13 & 0.06 & 0.33 & 0.13 & 0.04 & 0.01 & 0.27 & 0.12 & 0.04 & 0.01 & \\
\hline RMSD & 0.71 & 0.32 & 0.14 & 0.06 & 0.37 & 0.14 & 0.04 & 0.01 & 0.30 & 0.13 & 0.05 & 0.01 & \\
\hline MAX & 1.08 & 0.46 & 0.22 & 0.10 & 0.65 & 0.22 & 0.07 & 0.03 & 0.54 & 0.20 & 0.08 & 0.03 & \\
\hline
\end{tabular}

TABLE II. IPs (in eV) of the 20 smallest molecules of the GW100 set computed at the $G_{0} W_{0} @$ PBE0 level of theory with various basis sets and corrections. The mean absolute deviation (MAD), root-mean-square deviation (RMSD), and maximum deviation (MAX) with respect to the $G_{0} W_{0} @$ PBE0/CBS values are also reported.

\begin{tabular}{|c|c|c|c|c|c|c|c|c|c|c|c|c|c|}
\hline \multirow[b]{2}{*}{ Mol. } & \multicolumn{4}{|c|}{$G_{0} W_{0} @ \mathrm{PBE} 0$} & \multicolumn{4}{|c|}{$G_{0} W_{0} @$ PBE0+srLDA } & \multicolumn{4}{|c|}{$G_{0} W_{0} @ \mathrm{PBE} 0+\mathrm{srPBE}$} & \multirow{2}{*}{$\frac{G_{0} W_{0} @ \text { PBE0 }}{\text { CBS }}$} \\
\hline & cc-pVDZ & cc-pVTZ & cc-pVQZ & $\overline{c c-p V 5 Z}$ & $\overline{c c-p V D Z}$ & cc-pVTZ & cc-pVQZ & $\overline{c c-p V 5 Z}$ & cc-pVDZ & cc-pVTZ & cc-pVQZ & $\overline{c c-p V 5 Z}$ & \\
\hline $\mathrm{He}$ & 23.99 & 23.98 & 24.03 & 24.04 & 24.26 & 24.09 & 24.09 & 24.07 & 24.29 & 24.10 & 24.08 & 24.07 & 24.06 \\
\hline $\mathrm{Ne}$ & 20.35 & 20.88 & 21.05 & 21.05 & 20.86 & 21.16 & 21.22 & 21.16 & 21.05 & 21.22 & 21.23 & 21.15 & 21.12 \\
\hline $\mathrm{H}_{2}$ & 15.98 & 16.13 & 16.19 & 16.21 & 16.16 & 16.20 & 16.22 & 16.22 & 16.16 & 16.19 & 16.22 & 16.22 & 16.23 \\
\hline $\mathrm{Li}_{2}$ & 5.15 & 5.24 & 5.28 & 5.31 & 5.23 & 5.28 & 5.30 & 5.32 & 5.21 & 5.27 & 5.30 & 5.32 & 5.32 \\
\hline $\mathrm{LiH}$ & 7.32 & 7.49 & 7.56 & 7.59 & 7.48 & 7.55 & 7.59 & 7.61 & 7.45 & 7.54 & 7.58 & 7.61 & 7.62 \\
\hline $\mathrm{HF}$ & 14.95 & 15.61 & 15.82 & 15.85 & 15.41 & 15.85 & 15.97 & 15.94 & 15.56 & 15.89 & 15.97 & 15.93 & 15.94 \\
\hline $\mathrm{Ar}$ & 14.93 & 15.25 & 15.42 & 15.50 & 15.37 & 15.50 & 15.58 & 15.60 & 15.44 & 15.52 & 15.58 & 15.59 & 15.56 \\
\hline $\mathrm{H}_{2} \mathrm{O}$ & 11.53 & 12.21 & 12.43 & 12.47 & 11.95 & 12.43 & 12.55 & 12.54 & 12.05 & 12.45 & 12.55 & 12.54 & 12.56 \\
\hline $\mathrm{LiF}$ & 9.89 & 10.60 & 10.82 & 10.94 & 10.35 & 10.84 & 10.96 & 11.02 & 10.48 & 10.87 & 10.96 & 11.02 & 11.02 \\
\hline $\mathrm{HCl}$ & 11.96 & 12.34 & 12.50 & 12.57 & 12.35 & 12.56 & 12.64 & 12.65 & 12.39 & 12.56 & 12.63 & 12.64 & 12.63 \\
\hline $\mathrm{BeO}$ & 9.16 & 9.44 & 9.63 & 9.74 & 9.53 & 9.64 & 9.74 & 9.80 & 9.61 & 9.65 & 9.74 & 9.79 & 9.80 \\
\hline $\mathrm{CO}$ & 13.67 & 14.02 & 14.13 & 14.18 & 14.00 & 14.18 & 14.22 & 14.23 & 14.05 & 14.18 & 14.22 & 14.23 & 14.22 \\
\hline $\mathrm{N}_{2}$ & 14.84 & 15.30 & 15.44 & 15.50 & 15.22 & 15.50 & 15.55 & 15.56 & 15.31 & 15.51 & 15.54 & 15.55 & 15.55 \\
\hline $\mathrm{CH}_{4}$ & 13.85 & 14.15 & 14.27 & 14.30 & 14.11 & 14.27 & 14.32 & 14.33 & 14.15 & 14.27 & 14.32 & 14.33 & 14.35 \\
\hline $\mathrm{BH}_{3}$ & 12.87 & 13.13 & 13.22 & 13.26 & 13.09 & 13.23 & 13.27 & 13.28 & 13.10 & 13.22 & 13.26 & 13.28 & 13.29 \\
\hline $\mathrm{NH}_{3}$ & 9.96 & 10.56 & 10.73 & 10.75 & 10.31 & 10.72 & 10.82 & 10.80 & 10.37 & 10.72 & 10.81 & 10.79 & 10.82 \\
\hline $\mathrm{BF}$ & 10.66 & 10.87 & 10.92 & 10.94 & 10.88 & 10.96 & 10.97 & 10.97 & 10.88 & 10.95 & 10.96 & 10.97 & 10.96 \\
\hline $\mathrm{BN}$ & 11.07 & 11.40 & 11.54 & 11.60 & 11.40 & 11.56 & 11.63 & 11.65 & 11.45 & 11.56 & 11.62 & 11.65 & 11.65 \\
\hline $\mathrm{SH}_{2}$ & 9.69 & 10.10 & 10.25 & 10.30 & 10.03 & 10.28 & 10.35 & 10.36 & 10.04 & 10.27 & 10.34 & 10.35 & 10.36 \\
\hline $\mathrm{F}_{2}$ & 14.92 & 15.38 & 15.57 & 15.64 & 15.41 & 15.65 & 15.73 & 15.74 & 15.57 & 15.69 & 15.73 & 15.73 & 15.71 \\
\hline MAD & 0.60 & 0.24 & 0.10 & 0.05 & 0.29 & 0.07 & 0.02 & 0.01 & 0.23 & 0.07 & 0.03 & 0.01 & \\
\hline RMSD & 0.66 & 0.26 & 0.11 & 0.06 & 0.33 & 0.08 & 0.03 & 0.02 & 0.27 & 0.08 & 0.04 & 0.01 & \\
\hline MAX & 1.12 & 0.42 & 0.19 & 0.09 & 0.67 & 0.18 & 0.09 & 0.04 & 0.54 & 0.15 & 0.10 & 0.03 & \\
\hline
\end{tabular}

for the energy, ${ }^{104}$ and ii) because the present scheme only corrects the basis-set incompleteness error originating from the electron-electron cusp, some incompleteness remains at the HF or KS level. ${ }^{105}$

\section{B. Nucleobases}

In order to check the transferability of the present observations to larger systems, we have computed the values of the IPs of the five canonical nucleobases (adenine, cytosine, thymine, guanine, and uracil) at the $G_{0} W_{0} @$ PBE level of theory with a different basis set family. ${ }^{109,110}$ The numerical values are reported in Table III, and their error with respect to the $G_{0} W_{0} @ \mathrm{PBE} / \mathrm{def} 2-\mathrm{TQZVP}$ extrapolated values ${ }^{16}$ (obtained via extrapolation of the def2-TZVP and def2-QZVP results) are shown in Fig. 2. Table III also contains extrapolated IPs obtained with plane-wave basis sets with two different software packages. ${ }^{23,106}$ The CCSD(T)/def2-TZVPP computed by Krause et al. ${ }^{108}$ on the same geometries, the CCSD(T)//CCSD/aug-cc-pVDZ results from Ref. 107, as well as the experimental results extracted from Ref. 16 are reported 

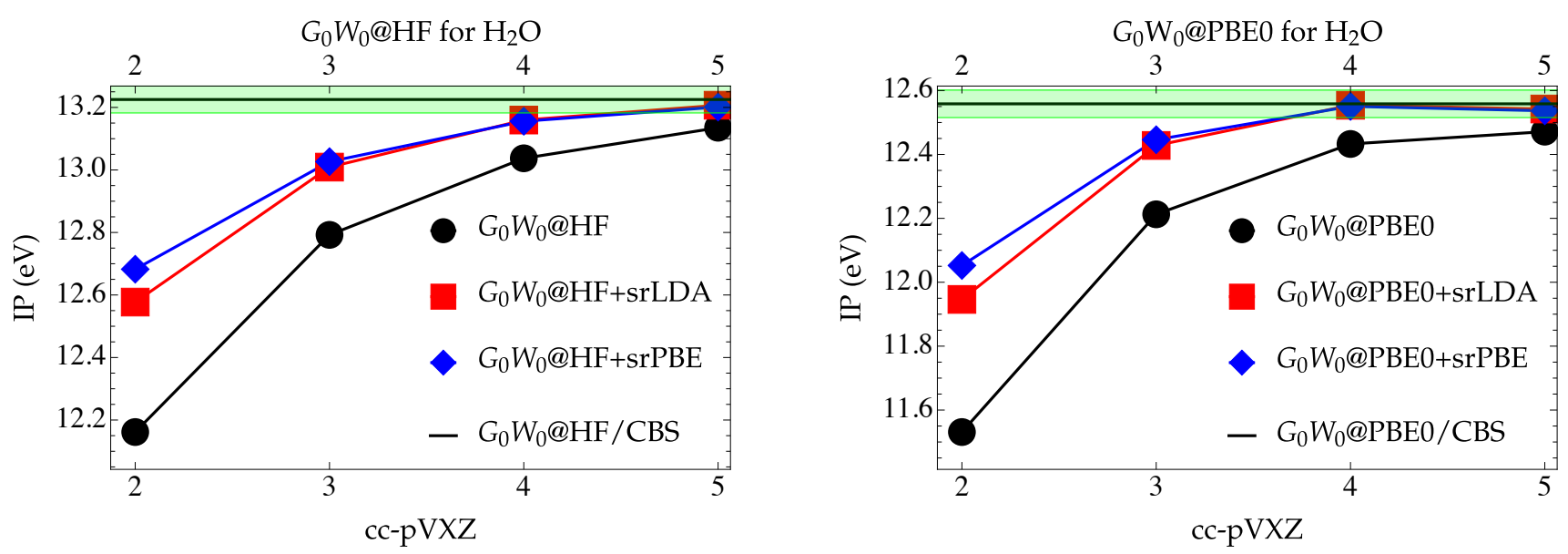

FIG. 1. IP (in eV) of the water molecule computed at the $G_{0} W_{0}$ (black circles), $G_{0} W_{0}+$ srLDA (red squares), and $G_{0} W_{0}+$ srPBE (blue diamonds) levels of theory with increasingly large Dunning's basis sets ${ }^{102}$ (cc-pVDZ, cc-pVTZ, cc-pVQZ, and cc-pV5Z) with two different starting points: HF (left) and PBE0 (right). The thick black line represents the CBS value obtained by extrapolation (see text for more details). The green area corresponds to chemical accuracy (i.e, error below $1 \mathrm{kcal} / \mathrm{mol}$ or $0.043 \mathrm{eV}$ ).

TABLE III. IPs (in eV) of the five canonical nucleobases (adenine, cytosine, thymine, guanine, and uracil) computed at the $G_{0} W_{0} @$ PBE level of theory for various basis sets and corrections. The deviation with respect to the $G_{0} W_{0} @ \mathrm{PBE} / \mathrm{def} 2-\mathrm{TQZVP}$ extrapolated values are reported in square brackets. The extrapolation error is reported in parenthesis. Extrapolated $G_{0} W_{0} @ \mathrm{PBE}$ results obtained with plane-wave basis sets, as well as $\operatorname{CCSD}(\mathrm{T})$ and experimental results are reported for comparison.

\begin{tabular}{|c|c|c|c|c|c|c|}
\hline \multirow[b]{2}{*}{ Method } & \multirow[b]{2}{*}{ Basis } & \multicolumn{5}{|c|}{ IPs of nucleobases $(\mathrm{eV})$} \\
\hline & & Adenine & Cytosine & Guanine & Thymine & Uracil \\
\hline$G_{0} W_{0} @ \mathrm{PBE}^{\mathrm{a}}$ & def2-SVP & $7.27[-0.88]$ & $7.53[-0.92]$ & $6.95[-0.92]$ & $8.02[-0.85]$ & $8.38[-1.00]$ \\
\hline$G_{0} W_{0} @ \mathrm{PBE}+\mathrm{srLDA}^{\mathrm{a}}$ & def2-SVP & $7.60[-0.55]$ & $7.95[-0.50]$ & $7.29[-0.59]$ & $8.36[-0.51]$ & $8.80[-0.58]$ \\
\hline$G_{0} W_{0} @ \mathrm{PBE}+\mathrm{srPBE}^{\mathrm{a}}$ & def2-SVP & $7.64[-0.51]$ & $8.06[-0.39]$ & $7.34[-0.54]$ & $8.41[-0.45]$ & $8.91[-0.47]$ \\
\hline$G_{0} W_{0} @ \mathrm{PBE}^{\mathrm{a}}$ & def2-TZVP & $7.74[-0.41]$ & $8.06[-0.39]$ & $7.45[-0.42]$ & $8.48[-0.38]$ & $8.86[-0.52]$ \\
\hline$G_{0} W_{0} @ \mathrm{PBE}+\mathrm{srLDA}^{\mathrm{a}}$ & def2-TZVP & $7.92[-0.23]$ & $8.26[-0.19]$ & $7.64[-0.23]$ & $8.67[-0.20]$ & $9.25[-0.13]$ \\
\hline$G_{0} W_{0} @ \mathrm{PBE}+\mathrm{srPBE}^{\mathrm{a}}$ & def2-TZVP & $7.92[-0.23]$ & $8.27[-0.18]$ & $7.64[-0.23]$ & $8.68[-0.19]$ & $9.27[-0.11]$ \\
\hline$G_{0} W_{0} @ \mathrm{PBE}^{\mathrm{b}}$ & def2-QZVP & $7.98[-0.18]$ & $8.29[-0.16]$ & $7.69[-0.18]$ & $8.71[-0.16]$ & $9.22[-0.16]$ \\
\hline$G_{0} W_{0} @ \mathrm{PBE}^{\mathrm{c}}$ & def2-TQZVP & $8.16(1)$ & $8.44(1)$ & $7.87(1)$ & $8.87(1)$ & $9.38(1)$ \\
\hline$\overline{G_{0} W_{0} @ \mathrm{PBE}^{\mathrm{d}}}$ & plane waves & 8.12 & 8.40 & 7.85 & 8.83 & 9.36 \\
\hline$G_{0} W_{0} @ \mathrm{PBE}^{\mathrm{e}}$ & plane waves & $8.09(2)$ & $8.40(2)$ & $7.82(2)$ & $8.82(2)$ & $9.19(2)$ \\
\hline $\operatorname{CCSD}(T)^{\mathrm{f}}$ & aug-cc-pVDZ & 8.40 & 8.76 & 8.09 & 9.04 & 9.43 \\
\hline $\operatorname{CCSD}(\mathrm{T})^{\mathrm{g}}$ & def2-TZVPP & 8.33 & 9.51 & 8.03 & 9.08 & 10.13 \\
\hline Experiment $^{\mathrm{h}}$ & & 8.48 & 8.94 & 8.24 & 9.20 & 9.68 \\
\hline
\end{tabular}

a This work.

${ }^{\mathrm{b}}$ Unpublished data taken from https://gw100.wordpress.com obtained with TURBOMOLE v7.0.

${ }^{\mathrm{c}}$ Extrapolated values obtained from the def2-TZVP and def2-QZVP values.

${ }^{\mathrm{d}}$ Extrapolated plane-wave results from Ref. 23 obtained with WEST.

${ }^{\mathrm{e}}$ Extrapolated plane-wave results from Ref. 106 obtained with VASP.

${ }^{\mathrm{f}} \mathrm{CCSD}(\mathrm{T}) / / \mathrm{CCSD} /$ aug-cc-pVDZ results from Ref. 107.

${ }^{g}$ Reference 108.

${ }^{\mathrm{h}}$ Experimental values are taken from Ref. 16 and correspond to vertical ionization energies.

for comparison purposes.

For these five systems, the IPs are all of the order of 8 or 9 $\mathrm{eV}$ with an amplitude of roughly $1 \mathrm{eV}$ between the smallest basis set (def2-SVP) and the CBS value. The conclusions that we have drawn in the previous subsection do apply here as well. For the smallest double- $\zeta$ basis def2-SVP, the basisset correction reduces by roughly half an $\mathrm{eV}$ the basis-set incompleteness error. It is particularly interesting to note that the basis-set corrected def2-TZVP results are on par with the correction-free def2-QZVP numbers. This is quite remarkable as the number of basis functions jumps from 371 to 777 for the largest system (guanine).

\section{CONCLUSION}

In the present manuscript, we have shown that the densitybased basis-set correction developed by some of the authors 


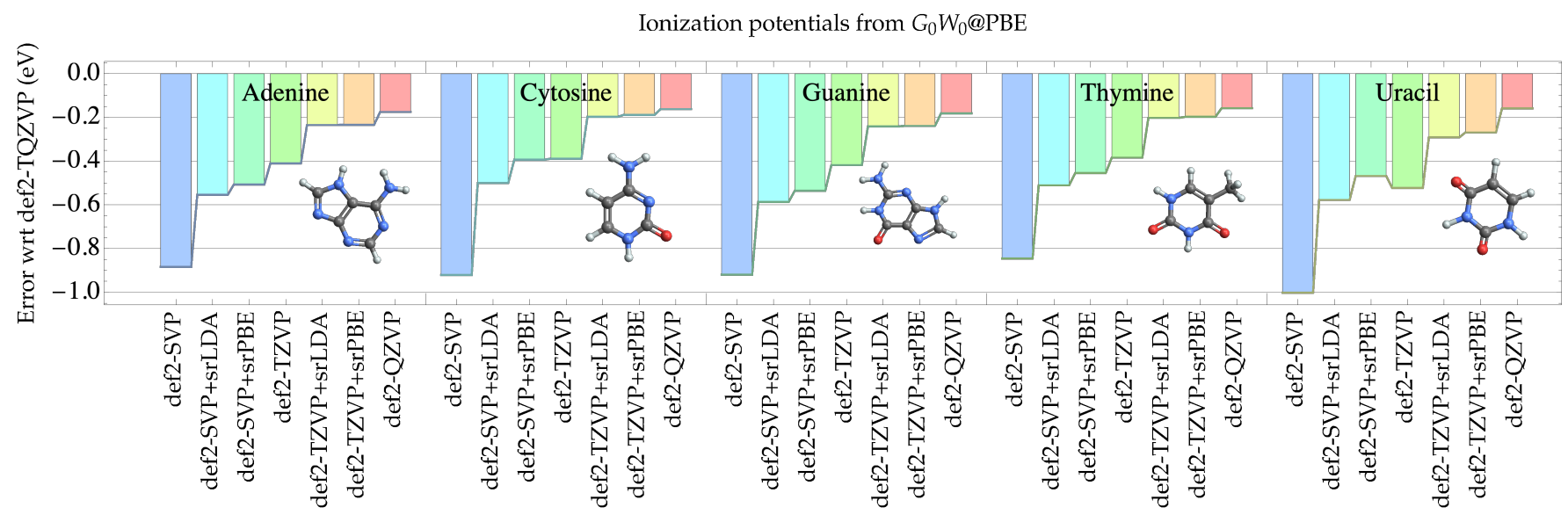

FIG. 2. Error (in eV) with respect to the $G_{0} W_{0} @ P B E / d e f 2-T Q Z V P$ extrapolated values for the IPs of the five canonical nucleobases (adenine, cytosine, thymine, guanine, and uracil) computed at the $G_{0} W_{0} @ \mathrm{PBE}$ level of theory for various basis sets and corrections.

in Ref. 56 and applied recently to ground- and excited-state properties $^{57,58}$ can also be successfully applied to Green function methods such as $G W$. In particular, we have evidenced that the present basis-set correction (which relies on LDA- or PBE-based short-range correlation functionals) significantly speeds up the convergence of IPs for small and larger molecules towards the CBS limit. These findings have been observed for different $G W$ starting points (HF, PBE, and PBE0). We have observed that the performance of the two short-range correlation functionals (srLDA and srPBE) are quite similar with a slight edge for srPBE over srLDA. Therefore, because srPBE is only slightly more computationally expensive than srLDA, we do recommend the use of srPBE.

As mentioned earlier, the present basis-set correction can be straightforwardly applied to other properties of interest such as electron affinities or fundamental gaps. It is also applicable to other flavors of $G W$ such as the partially self-consistent evGW or qs $G W$ methods, and more generally to any approximation of the self-energy. We are currently investigating the performance of the present approach within linear response theory in order to speed up the convergence of excitation energies obtained within the RPA and Bethe-Salpeter equation (BSE) $)^{24,111,112}$ formalisms. We hope to report on this in the near future.

\section{SUPPORTING INFORMATION}

See supporting information for the expression of the shortrange correlation potentials, additional graphs reporting the convergence of the ionization potentials of the GW20 subset with respect to the size of the basis set, and the numerical data of Tables I and II (provided in txt and json formats).

\section{ACKNOWLEDGMENTS}

PFL would like to thank Fabien Bruneval for technical assistance. PFL and JT would like to thank Arjan Berger and Pina Romaniello for stimulating discussions. This work was performed using HPC resources from GENCI-TGCC (Grant No. 2018-A0040801738) and CALMIP (Toulouse) under allocation 2019-18005. Funding from the "Centre National de la Recherche Scientifique” is acknowledged. This work has been supported through the EUR grant NanoX ANR-17-EURE0009 in the framework of the "Programme des Investissements d'Avenir".

\footnotetext{
${ }^{1}$ Martin, R. M.; Reining, L.; Ceperley, D. M. Interacting Electrons: Theory and Computational Approaches; Cambridge University Press, 2016.

${ }^{2}$ Aryasetiawan, F.; Gunnarsson, O. The GW method. Rep. Prog. Phys. 1998, 61, 237-312.

${ }^{3}$ Onida, G.; Reining, L.; and, A. R. Electronic excitations: density-functional versus many-body Green's-function approaches. Rev. Mod. Phys. 2002, 74, 601-659.

${ }^{4}$ Reining, L. The GW Approximation: Content, Successes and Limitations: The GW Approximation. Wiley Interdiscip. Rev. Comput. Mol. Sci. 2017, e1344.

${ }^{5}$ Blase, X.; Attaccalite, C.; Olevano, V. First-Principles GW Calculations for Fullerenes, Porphyrins, Phtalocyanine, and Other Molecules of Interest for Organic Photovoltaic Applications. Phys. Rev. B 2011, 83, 115103.

${ }^{6}$ Faber, C.; Attaccalite, C.; Olevano, V.; Runge, E.; Blase, X. First-Principles GW Calculations for DNA and RNA Nucleobases. Phys. Rev. B 2011, 83, 115123.

${ }^{7}$ Bruneval, F. Ionization Energy of Atoms Obtained from $G W$ Self-Energy or from Random Phase Approximation Total Energies. J. Chem. Phys. 2012, 136, 194107.

${ }^{8}$ Bruneval, F.; Hamed, S. M.; Neaton, J. B. A Systematic Benchmark of the Ab Initio Bethe-Salpeter Equation Approach for Low-Lying Optical Excitations of Small Organic Molecules. J. Chem. Phys. 2015, 142, 244101. ${ }^{9}$ Bruneval, F.; Rangel, T.; Hamed, S. M.; Shao, M.; Yang, C.; Neaton, J. B. Molgw 1: Many-Body Perturbation Theory Software for Atoms, Molecules, and Clusters. Comput. Phys. Commun. 2016, 208, 149-161.

${ }^{10}$ Bruneval, F. Optimized Virtual Orbital Subspace for Faster GW Calculations in Localized Basis. J. Chem. Phys. 2016, 145, 234110.

${ }^{11}$ Boulanger, P.; Jacquemin, D.; Duchemin, I.; Blase, X. Fast and Accurate Electronic Excitations in Cyanines with the Many-Body Bethe-Salpeter Approach. J. Chem. Theory Comput. 2014, 10, 1212-1218.

${ }^{12}$ Blase, X.; Boulanger, P.; Bruneval, F.; Fernandez-Serra, M.; Duchemin, I. $G W$ and Bethe-Salpeter Study of Small Water Clusters. J. Chem. Phys. 2016, 144, 034109.

${ }^{13}$ Li, J.; Holzmann, M.; Duchemin, I.; Blase, X.; Olevano, V. Helium Atom Excitations by the G W and Bethe-Salpeter Many-Body Formalism. Phys. Rev. Lett. 2017, 118, 163001.

${ }^{14}$ Hung, L.; da Jornada, F. H.; Souto-Casares, J.; Chelikowsky, J. R.; Louie, S. G.; Öğüt, S. Excitation Spectra of Aromatic Molecules within a
} 
Real-Space G W -BSE Formalism: Role of Self-Consistency and Vertex Corrections. Phys. Rev. B 2016, 94, 085125.

${ }^{15}$ Hung, L.; Bruneval, F.; Baishya, K.; Öğüt, S. Benchmarking the $G W$ Approximation and Bethe-Salpeter Equation for Groups IB and IIB Atoms and Monoxides. J. Chem. Theory Comput. 2017, 13, 2135-2146.

${ }^{16}$ van Setten, M. J.; Caruso, F.; Sharifzadeh, S.; Ren, X.; Scheffler, M.; Liu, F.; Lischner, J.; Lin, L.; Deslippe, J. R.; Louie, S. G.; Yang, C.; Weigend, F.; Neaton, J. B.; Evers, F.; Rinke, P. $G W$ 100: Benchmarking $G_{0} W_{0}$ for Molecular Systems. J. Chem. Theory Comput. 2015, 11, 5665-5687.

${ }^{17}$ van Setten, M. J.; Costa, R.; Viñes, F.; Illas, F. Assessing $G W$ Approaches for Predicting Core Level Binding Energies. J. Chem. Theory Comput. 2018, 14, 877-883.

${ }^{18} \mathrm{Ou}$, Q.; Subotnik, J. E. Comparison between $G W$ and Wave-Function-Based Approaches: Calculating the Ionization Potential and Electron Affinity for 1D Hubbard Chains. J. Phys. Chem. A 2016, 120, 4514-4525.

${ }^{19} \mathrm{Ou}, \mathrm{Q} . ;$ Subotnik, J. E. Comparison between the Bethe-Salpeter Equation and Configuration Interaction Approaches for Solving a Quantum Chemistry Problem: Calculating the Excitation Energy for Finite 1D Hubbard Chains. J. Chem. Theory Comput. 2018, 14, 527-542.

${ }^{20}$ Faber, C. Electronic, Excitonic and Polaronic Properties of Organic Systems within the Many-Body GW and Bethe-Salpeter Formalisms: Towards Organic Photovoltaics. PhD Thesis, Université de Grenoble, 2014.

${ }^{21}$ Marini, A.; Hogan, C.; Gruning, M.; Varsano, D. Yambo: An Ab Initio Tool For Excited State Calculations. Comp. Phys. Comm. 2009, 180, 1392

${ }^{22}$ Deslippe, J.; Samsonidze, G.; Strubbe, D. A.; Jain, M.; Cohen, M. L.; Louie, S. G. BerkeleyGW: A Massively Parallel Computer Package for the Calculation of the Quasiparticle and Optical Properties of Materials and Nanostructures. Comput. Phys. Commun. 2012, 183, 1269.

${ }^{23}$ Maggio, E.; Liu, P.; van Setten, M. J.; Kresse, G. GW 100: A Plane Wave Perspective for Small Molecules. J. Chem. Theory Comput. 2017, 13, 635648.

${ }^{24}$ Blase, X.; Duchemin, I.; Jacquemin, D. The Bethe-Salpeter Equation in Chemistry: Relations with TD-DFT, Applications and Challenges. Chem. Soc. Rev. 2018, 47, 1022-1043.

${ }^{25}$ van Setten, M. J.; Weigend, F.; Evers, F. The $G W$-Method for Quantum Chemistry Applications: Theory and Implementation. J. Chem. Theory Comput. 2013, 9, 232-246.

${ }^{26}$ Kaplan, F.; Weigend, F.; Evers, F.; van Setten, M. J. Off-Diagonal SelfEnergy Terms and Partially Self-Consistency in $G W$ Calculations for Single Molecules: Efficient Implementation and Quantitative Effects on Ionization Potentials. J. Chem. Theory Comput. 2015, 11, 5152-5160.

${ }^{27}$ Kaplan, F.; Harding, M. E.; Seiler, C.; Weigend, F.; Evers, F.; van Setten, M. J. Quasi-Particle Self-Consistent $G W$ for Molecules. J. Chem. Theory Comput. 2016, 12, 2528-2541.

${ }^{28}$ Krause, K.; Klopper, W. Implementation Of The Bethe-Salpeter Equation In The Turbomole Program. J. Comput. Chem. 2017, 38, 383-388.

${ }^{29}$ Caruso, F.; Rinke, P.; Ren, X.; Scheffler, M.; Rubio, A. Unified Description of Ground and Excited States of Finite Systems: The Self-Consistent G W Approach. Phys. Rev. B 2012, 86, 081102(R).

${ }^{30}$ Caruso, F.; Rohr, D. R.; Hellgren, M.; Ren, X.; Rinke, P.; Rubio, A.; Scheffler, M. Bond Breaking and Bond Formation: How Electron Correlation Is Captured in Many-Body Perturbation Theory and Density-Functional Theory. Phys. Rev. Lett. 2013, 110, 146403.

${ }^{31}$ Caruso, F.; Rinke, P.; Ren, X.; Rubio, A.; Scheffler, M. Self-Consistent G W : All-Electron Implementation with Localized Basis Functions. Phys. Rev. B 2013, 88, 075105.

${ }^{32}$ Caruso, F. Self-Consistent GW Approach for the Unified Description of Ground and Excited States of Finite Systems. PhD Thesis, Freie Universität Berlin, 2013.

${ }^{33}$ Hedin, L. New Method for Calculating the One-Particle Green's Function with Application to the Electron-Gas Problem. Phys. Rev. 1965, 139, A796.

${ }^{34}$ Olver, F. W. J., Lozier, D. W., Boisvert, R. F., Clark, C. W., Eds. NIST Handbook of Mathematical Functions; Cambridge University Press: New York, 2010.

${ }^{35}$ Loos, P. F.; Romaniello, P.; Berger, J. A. Green functions and selfconsistency: insights from the spherium model. J. Chem. Theory Comput. 2018, 14, 3071-3082.

${ }^{36}$ Hybertsen, M. S.; Louie, S. G. First-Principles Theory of Quasiparticles: Calculation of Band Gaps in Semiconductors and Insulators. Phys. Rev. Lett. 1985, 55, 1418-1421.
${ }^{37}$ Hybertsen, M. S.; Louie, S. G. Electron Correlation in Semiconductors and Insulators: Band Gaps and Quasiparticle Energies. Phys. Rev. B 1986, 34 , 5390-5413.

${ }^{38}$ Bruneval, F.; Marques, M. A. L. Benchmarking the Starting Points of the GW Approximation for Molecules. J. Chem. Theory Comput. 2013, 9, 324-329.

${ }^{39}$ Jacquemin, D.; Duchemin, I.; Blase, X. Assessment Of The Convergence Of Partially Self-Consistent BSE/GW Calculations. Mol. Phys. 2016, 114, 957.

${ }^{40}$ Gui, X.; Holzer, C.; Klopper, W. Accuracy Assessment of GW Starting Points for Calculating Molecular Excitation Energies Using the Bethe-Salpeter Formalism. J. Chem. Theory Comput. 2018, 14, 2127-2136.

${ }^{41}$ Shishkin, M.; Kresse, G. Self-Consistent G W Calculations for Semiconductors and Insulators. Phys. Rev. B 2007, 75, 235102.

${ }^{42} \mathrm{Ke}$, S.-H. All-Electron G W Methods Implemented in Molecular Orbital Space: Ionization Energy and Electron Affinity of Conjugated Molecules. Phys. Rev. B 2011, 84, 205415.

${ }^{43}$ Koval, P.; Foerster, D.; Sánchez-Portal, D. Fully Self-Consistent G W and Quasiparticle Self-Consistent G W for Molecules. Phys. Rev. B 2014, 89 , 155417.

${ }^{44}$ Jacquemin, D.; Duchemin, I.; Blondel, A.; Blase, X. Benchmark of BetheSalpeter for Triplet Excited-States. J. Chem. Theory Comput. 2017, 13, 767-783.

${ }^{45}$ Kato, T. On The Eigenfunctions Of Many-Particle Systems In Quantum Mechanics. Commun. Pure Appl. Math. 1957, 10, 151.

${ }^{46}$ Hylleraas, E. A. Neue Berechnung der Energie des Heliums im Grundzustande, sowie des tiefsten Terms von Ortho-Helium. Z. Phys. 1929, 54, 347.

${ }^{47}$ Kutzelnigg, W. R12-Dependent Terms In The Wave Function As Closed Sums Of Partial Wave Amplitudes For Large L. Theor. Chim. Acta 1985 , $68,445$.

${ }^{48}$ Noga, J.; Kutzelnigg, W. Coupled Cluster Theory That Takes Care Of The Correlation Cusp By Inclusion Of Linear Terms In The Interelectronic Coordinates. J. Chem. Phys. 1994, 101, 7738.

${ }^{49}$ Kutzelnigg, W.; Klopper, W. Wave Functions With Terms Linear In The Interelectronic Coordinates To Take Care Of The Correlation Cusp. I. General Theory. J. Chem. Phys. 1991, 94, 1985.

${ }^{50}$ Kong, L.; Bischo, F. A.; Valeev, E. F. Explicitly Correlated R12/F12 Methods for Electronic Structure. Chem. Rev. 2012, 112, 75.

${ }^{51}$ Hattig, C.; Klopper, W.; Kohn, A.; Tew, D. P. Explicitly Correlated Electrons in Molecules. Chem. Rev. 2012, 112, 4.

${ }^{52}$ Ten-no, S.; Noga, J. Explicitly Correlated Electronic Structure Theory From R12/F12 Ansatze. WIREs Comput. Mol. Sci. 2012, 2, 114.

${ }^{53}$ Ten-no, S. Explicitly Correlated Wave Functions: Summary And Perspective. Theor. Chem. Acc. 2012, 131, 1070.

${ }^{54}$ Grüneis, A.; Hirata, S.; Ohnishi, Y.-Y.; Ten-no, S. Perspective: Explicitly Correlated Electronic Structure Theory For Complex Systems. J. Chem. Phys. 2017, 146, 080901.

${ }^{55}$ Tew, D. P.; Klopper, W.; Neiss, C.; Hattig, C. Quintuple- $\zeta$ Quality CoupledCluster Correlation Energies With Triple- $\zeta$ Basis Sets. Phys. Chem. Chem. Phys. 2007, 9, 1921.

${ }^{56}$ Giner, E.; Pradines, B.; Ferté, A.; Assaraf, R.; Savin, A.; Toulouse, J. Curing Basis-Set Convergence Of Wave-Function Theory Using DensityFunctional Theory: A Systematically Improvable Approach. J. Chem. Phys. 2018, 149, 194301.

${ }^{57}$ Loos, P. F.; Pradines, B.; Scemama, A.; Toulouse, J.; Giner, E. A DensityBased Basis-Set Correction for Wave Function Theory. J. Phys. Chem. Lett. 2019, 10, 2931-2937.

${ }^{58}$ Giner, E.; Scemama, A.; Toulouse, J.; Loos, P. F. Chemically Accurate Excitation Energies With Small Basis Sets. J. Chem. Phys. 2019, 151, 144118.

${ }^{59}$ Barca, G. M. J.; Loos, P.-F.; Gill, P. M. W. Many-Electron Integrals over Gaussian Basis Functions. I. Recurrence Relations for Three-Electron Integrals. Journal of Chemical Theory and Computation 2016, 12, 1735-1740.

${ }^{60}$ Barca, G. M.; Loos, P.-F. Three-and Four-Electron Integrals Involving Gaussian Geminals: Fundamental Integrals, Upper Bounds, and Recurrence Relations. The Journal of chemical physics 2017, 147, 024103.

${ }^{61}$ Barca, G. M.; Loos, P.-F. Recurrence Relations for Four-Electron Integrals Over Gaussian Basis Functions. In Advances in Quantum Chemistry; Elsevier, 2018; Vol. 76; pp 147-165. 
${ }^{62}$ Szabo, A.; Ostlund, N. S. Modern quantum chemistry; McGraw-Hill: New York, 1989.

${ }^{63}$ Casida, M. E.; Chong, D. P. Physical Interpretation and Assessment of the Coulomb-Hole and Screened-Exchange Approximation for Molecules. Phys. Rev. A 1989, 40, 4837-4848.

${ }^{64}$ Casida, M. E.; Chong, D. P. Simplified Green-Function Approximations: Further Assessment of a Polarization Model for Second-Order Calculation of Outer-Valence Ionization Potentials in Molecules. Phys. Rev. A 1991, 44, $5773-5783$

${ }^{65}$ Stefanucci, G.; van Leeuwen, R. Nonequilibrium Many-Body Theory of Quantum Systems: A Modern Introduction; Cambridge University Press: Cambridge, 2013.

${ }^{66}$ Ortiz, J. V. Electron Propagator Theory: An Approach to Prediction and Interpretation in Quantum Chemistry: Electron Propagator Theory. Wiley Interdiscip. Rev. Comput. Mol. Sci. 2013, 3, 123-142.

${ }^{67}$ Phillips, J. J.; Zgid, D. Communication: The Description of Strong Correlation within Self-Consistent Green's Function Second-Order Perturbation Theory. J. Chem. Phys. 2014, 140, 241101.

${ }^{68}$ Phillips, J. J.; Kananenka, A. A.; Zgid, D. Fractional Charge and Spin Errors in Self-Consistent Green's Function Theory. J. Chem. Phys. 2015, $142,194108$.

${ }^{69}$ Rusakov, A. A.; Phillips, J. J.; Zgid, D. Local Hamiltonians for Quantitative Green's Function Embedding Methods. J. Chem. Phys. 2014, 141, 194105

${ }^{70}$ Rusakov, A. A.; Zgid, D. Self-Consistent Second-Order Green's Function Perturbation Theory for Periodic Systems. J. Chem. Phys. 2016, 144, 054106.

${ }^{71}$ Hirata, S.; Hermes, M. R.; Simons, J.; Ortiz, J. V. General-Order ManyBody Green's Function Method. J. Chem. Theory Comput. 2015, 11, 15951606.

${ }^{72}$ Hirata, S.; Doran, A. E.; Knowles, P. J.; Ortiz, J. V. One-Particle ManyBody Green's Function Theory: Algebraic Recursive Definitions, LinkedDiagram Theorem, Irreducible-Diagram Theorem, and General-Order Algorithms. J. Chem. Phys. 2017, 147, 044108.

${ }^{73}$ Ohnishi, Y.-y.; Ten-no, S. Explicitly Correlated Frequency-Independent Second-Order Green's Function for Accurate Ionization Energies. J. Comput. Chem. 2016, 37, 2447-2453.

${ }^{74}$ Johnson, C. M.; Doran, A. E.; Ten-no, S. L.; Hirata, S. Monte Carlo Explicitly Correlated Many-Body Green's Function Theory. J. Chem. Phys. 2018, 149, 174112.

${ }^{75}$ Pavošević, F.; Peng, C.; Ortiz, J. V.; Valeev, E. F. Communication: Explicitly Correlated Formalism for Second-Order Single-Particle Green's Function. J. Chem. Phys. 2017, 147, 121101

${ }^{76}$ Teke, N. K.; Pavosevic, F.; Peng, C.; Valeev, E. F. Explicitly Correlated Renormalized Second-Order Green's Function For Accurate Ionization Potentials Of Closed-Shell Molecules. J. Chem. Phys. 2019, 150, 214103.

${ }^{77}$ Levy, M. Universal Variational Functionals Of Electron Densities, FirstOrder Density Matrices, And Natural Spin-Orbitals And Solution Of The V-Representability Problem. Proc. Natl. Acad. Sci. U.S.A. 1979, 76, 6062.

${ }^{78}$ Levy, M. Electron Densities In Search Of Hamiltonians. Phys. Rev. A 1982, 26, 1200.

${ }^{79}$ Lieb, E. H. Density Functionals For Coulomb Systems. Int. J. Quantum Chem. 1983, 24, 243.

${ }^{80}$ Dahlen, N. E.; van Leeuwen, R. Self-Consistent Solution of the Dyson Equation for Atoms and Molecules within a Conserving Approximation. $J$. Chem. Phys. 2005, 122, 164102.

${ }^{81}$ Dahlen, N. E.; Van Leeuwen, R.; Von Barth, U. Variational Energy Functionals of the Green Function Tested on Molecules. Int. J. Quantum Chem. 2005, 101, 512-519.

${ }^{82}$ Dahlen, N. E.; van Leeuwen, R.; von Barth, U. Variational Energy Functionals of the Green Function and of the Density Tested on Molecules. Phys. Rev. A 2006, 73, 012511 .

${ }^{83}$ Gill, P. M. W. Molecular Integrals Over Gaussian Basis Functions. Adv. Quantum Chem. 1994, 25, 141-205.

${ }^{84}$ Casida, M. E. Generalization of the Optimized-Effective-Potential Model to Include Electron Correlation: A Variational Derivation of the ShamSchlüter Equation for the Exact Exchange-Correlation Potential. Phys. Rev. A 1995, 51, 2005-2013.

${ }^{85}$ Dreuw, A.; Head-Gordon, M. Single-Reference Ab Initio Methods for the Calculation of Excited States of Large Molecules. Chem. Rev. 2005, 105, 4009-4037.
${ }^{86}$ Veril, M.; Romaniello, P.; Berger, J. A.; Loos, P. F. Unphysical Discontinuities in GW Methods. J. Chem. Theory Comput. 2018, 14, 5220.

${ }^{87}$ Martin, P. C.; Schwinger, J. Theory of Many-Particle Systems. I. Phys. Rev. 1959, 115, 1342-1373.

${ }^{88}$ Baym, G.; Kadanoff, L. P. Conservation Laws and Correlation Functions. Phys. Rev. 1961, 124, 287-299.

${ }^{89}$ Baym, G. Self-Consistent Approximations in Many-Body Systems. Phys. Rev. 1962, 127, 1391-1401

${ }^{90}$ von Barth, U.; Holm, B. Self-Consistent GW 0 Results for the Electron Gas: Fixed Screened Potential W 0 within the Random-Phase Approximation. Phys. Rev. B 1996, 54, 8411 .

${ }^{91}$ Toulouse, J.; Gori-Giorgi, P.; Savin, A. A Short-Range Correlation Energy Density Functional With Multi-Determinantal Reference. Theor. Chem. Acc. 2005, 114, 305 .

${ }^{92}$ Paziani, S.; Moroni, S.; Gori-Giorgi, P.; Bachelet, G. B. Local-Spin-Density Functional For Multideterminant Density Functional Theory. Phys. Rev. B 2006, 73, 155111.

${ }^{93}$ Loos, P.-F.; Gill, P. M. W. The Uniform Electron Gas. Wiley Interdiscip. Rev. Comput. Mol. Sci. 2016, 6, 410-429.

${ }^{94}$ Ferté, A.; Giner, E.; Toulouse, J. Range-Separated Multideterminant Density-Functional Theory With A Short-Range Correlation Functional Of The On-Top Pair Density. J. Chem. Phys. 2019, 150, 084103.

${ }^{95}$ Perdew, J. P.; Burke, K.; Ernzerhof, M. Generalized Gradient Approximation Made Simple. Phys. Rev. Lett. 1996, 77, 3865-3868.

${ }^{96}$ Toulouse, J.; Colonna, F.; Savin, A. Long-Range-Short-Range Separation Of The Electron-Electron Interaction In Density-Functional Theory. Phys. Rev. A 2004, 70, 062505.

${ }^{97}$ Gori-Giorgi, P.; Savin, A. Properties Of Short-Range And Long-Range Correlation Energy Density Functionals From Electron-Electron Coalescence. Phys. Rev. A 2006, 73, 032506.

${ }^{98}$ Garniron, Y.; Gasperich, K.; Applencourt, T.; Benali, A.; Ferté, A.; Paquier, J.; Pradines, B.; Assaraf, R.; Reinhardt, P.; Toulouse, J.; Barbaresco, P.; Renon, N.; David, G.; Malrieu, J. P.; Véril, M.; Caffarel, M.; Loos, P. F.; Giner, E.; Scemama, A. Quantum Package 2.0: A Open-Source Determinant-Driven Suite Of Programs. J. Chem. Theory Comput. 2019 15,3591 .

${ }^{99}$ Duchemin, I.; Li, J.; Blase, X. Hybrid and Constrained Resolution-ofIdentity Techniques for Coulomb Integrals. J. Chem. Theory Comput. 2017, $13,1199$.

${ }^{100}$ Rojas, H. N.; Godby, R. W.; Needs, R. J. Space-Time Method for Ab Initio Calculations of Self-Energies and Dielectric Response Functions of Solids Phys. Rev. Lett. 1995, 74, 1827.

${ }^{101}$ Duchemin, I.; Blase, X. Separable Resolution-of-the-Identity with AllElectron Gaussian Bases: Application to Cubic-scaling RPA. J. Chem. Phys. 2019, 150, 174120

${ }^{102}$ Dunning, T. H. Gaussian Basis Sets For Use In Correlated Molecular Calculations. I. The Atoms Boron Through Neon And Hydrogen. J. Chem. Phys. 1989, 90, 1007.

${ }^{103}$ Lewis, A. M.; Berkelbach, T. C. Vertex Corrections to the Polarizability Do Not Improve the GW Approximation for the Ionization Potential of Molecules. J. Chem. Theory Comput. 2019, 15, 2925.

${ }^{104}$ Kim, M.; Sim, E.; Burke, K. Understanding and Reducing Errors in Density Functional Calculations. Phys. Rev. Lett. 2013, 111, 073003.

${ }^{105}$ Adler, T. B.; Knizia, G.; Werner, H.-J. A Simple and Efficient CCSD(T)-F12 Approximation. J. Chem. Phys. 2007, 127, 221106.

${ }^{106}$ Govoni, M.; Galli, G. GW100: Comparison of Methods and Accuracy of Results Obtained with the WEST Code. J. Chem. Theory Comput. 2018 , 14, 1895-1909.

${ }^{107}$ Roca-Sanjuan, D.; Rubio, M.; Merchan, M.; Serrano-Andres, L. Ab Initio Determination of The Ionization Potentials of DNA And RNA Nucleobases. J. Chem. Phys. 2006, 125, 084302.

${ }^{108}$ Krause, K.; Harding, M. E.; Klopper, W. Coupled-Cluster Reference Values For The Gw27 And Gw100 Test Sets For The Assessment Of Gw Methods. Mol. Phys. 2015, 113, 1952.

${ }^{109}$ Weigend, F.; Furche, F.; Ahlrichs, R. Gaussian basis sets of quadruple zeta valence quality for atoms H-Kr. J. Chem. Phys. 2003, 119.

${ }^{110}$ Weigend, F.; Ahlrichs, R. Balanced basis sets of split valence, triple zeta valence and quadruple zeta valence quality for $\mathrm{H}$ to $\mathrm{Rn}$ : Design and assessment of accuracy. Phys. Chem. Chem. Phys. 2005, 7. 
${ }^{111}$ Strinati, G. Application of the Green's Functions Method to the Study of the Optical Properties of Semiconductors. Riv. Nuovo Cimento 1988, 11, $1-86$.
${ }^{112}$ Leng, X.; Jin, F.; Wei, M.; Ma, Y. GW Method and Bethe-Salpeter Equation for Calculating Electronic Excitations: GW Method and Bethe-Salpeter Equation. Wiley Interdiscip. Rev. Comput. Mol. Sci. 2016, 6, 532-550. 